\title{
PARASITIC ZOONOSES AND CAMEL
}

Muhammad Adeel Hassan ${ }^{1^{*}}$, Alireza Sazmand ${ }^{2,3}$, Abdullah Saghir Ahmad ${ }^{1}$, Muhammad Shehzad Hassan ${ }^{4}$, Muhammad Rashid $^{5}$, Syed Qaswar Ali Shah ${ }^{6}$ and Muhammad Mazhar Ayaz ${ }^{1}$

${ }^{1}$ Department of Parasitology, Cholistan University of Veterinary and Animal Sciences, Bahawalpur, Pakistan

${ }^{2}$ Department of Pathobiology, Faculty of Veterinary Science, Bu-Ali Sina University, Hamedan, Iran

3Zoonotic Diseases Research Center, School of Public Health, Shahid Sadoughi University of Medical Sciences, Yazd, Iran ${ }^{4}$ Department of Prosthetics and Orthotics, College of Medical Rehabilitation, Taibah University, Medinah, Saudi Arabia ${ }^{5}$ Department of Parasitology, Faculty of Veterinary and Animal Sciences, The Islamia University of Bahawalpur, Pakistan ${ }^{6}$ Department of Zoology, Cholistan University of Veterinary and Animal Sciences, Bahawalpur, Pakistan

*Corresponding author: adeelalvi21@yahoo.com

\section{INTRODUCTION}

With a total population of over 37 million heads, camels serve as an important source of milk and meat around the globe, especially in Asia and Africa. The single-humped camels, renowned as dromedary (Camelus dromedarius), are approximately $95 \%$ of the total population of camelids and are present in 47 countries (Food Agriculture Organization of The United Nations 2019), where they are playing a pivotal economic role. As the camels are an important food source in arid and semi-arid zones, the term used for camelids has been transformed from "ship of the desert" to "food security livestock" species. One evidence is that between the years 2008 and 2018, the camel world population increased by $21 \%$ compared to 4 , 5 , 9, and $15 \%$ for pigs, cattle, sheep, and goats, respectively (Food and Agriculture Organization of The United Nations 2019). However, despite being extremely resistant to harsh environmental conditions, dromedaries can get infected with several zoonotic pathogens, thus posing a public health risk (Sazmand et al. 2019b; Zhu et al. 2019).

Almost $65 \%$ of the published research articles on zoonotic pathogens of camels during 1970-2018 focused on Rift Valley fever, brucellosis, hydatidosis and Middle East respiratory syndrome (MERS) (Zhu et al. 2019). Despite, Echinococcosis in camels, which is the most studied zoonotic parasitic infection, some other parasites like Linguatula serrate, Trichinella spp., Fasciola spp., Cryptosporidium spp. and Toxoplasma gondii originating from camels cannot be ignored in view of public health significance (Zhu et al. 2019). Relatively a few parasites of camelids are considered as host specific (Schuster 2018), whereas most of the parasites infecting camels are i) nonzoonotic but with a large host range, or, ii) of zoonotic concern. Transmission routes of zoonotic parasites includes faecal contamination (Enterocytozoon spp., Blastocystis spp., Balantidium coli, Giardia duodenalis, Cryptosporidium spp.) or eating of undercooked meat or drinking raw infected milk (e.g., Linguatula serrate, Trichinella spp. and Toxoplasma gondii). In addition, camels act as reservoir for vector-borne protozoan parasites e.g. Trypanosoma evansi, gastropod-borne trematodes (e.g. Schistosoma spp., Dicrocoelium dendriticum and Fasciola spp.) or larvae of zoonotic cestodes, e.g. Echinococcus granulosus (sensu lato) (Sazmand and Joachim 2017; Sazmand et al. 2019b). Moreover, camels are the source blood for several haematophagous ectoparasites, such as ticks and fleas, which ultimately transmit zoonotic viral and bacterial pathogens e.g., Crimean-Congo hemorrhagic fever virus, Coxiella burnetii, Rickettsia spp., Bartonella spp. and Yersinia pestis (Wernery et al. 2014; Sazmand et al. 2019a;). In this chapter most important parasitic zoonoses related to camels are presented.

\section{Toxoplasmosis}

Toxoplasmosis is caused by Toxoplasma gondii, which is an intracellular protozoan that infects many domesticated and wild animals, as well as humans (Donahoe et al. 2015). Toxoplasma gondii was first discovered in 1908 and its life cycle was completely explained in the 1970s (Dubey and Frenkel 1972). Due to wide range of host species, T. gondii is one of the most important zoonotic parasites of the world (DjurkovićDjaković et al. 2019). About one third of the world's population is under the threat of this food and water borne parasite (Bahia-Oliveira et al. 2017). Previous studies have shown that 32,700 disability-adjusted life years (DALYs) of Toxoplasmosis are being reported annually in USA with 86,700 confirmed patients and 330 deaths (Scallan et al. 2015). However, its economic significance in developing and underdeveloped countries may be estimated to be much higher due to low level of food hygiene conditions.

The only reported definitive hosts for this parasite are the members of Felidae family (especially cats). Faeces of cats have unsporulated oocysts. Although these oocysts usually shed in feces for a few weeks, their large number makes them significant. These oocysts usually sporulate in 1-5 days in the environment and finally become infective. The intermediate hosts, including rodents and birds, become infected following the ingestion of soil, plant material or water contaminated with oocysts. Shortly after the ingestion, these oocysts transform into tachyzoites. The tachyzoites are settled in muscle and neural tissues and transform into bradyzoites. The cats get infected while consuming these intermediate hosts 
sheltering tissue cyst bradyzoites or by ingesting the sporulated oocysts directly. Camels however, can only get infected by ingesting the sporulated oocysts. Humans can become infected by any of the following routes:

- $\quad$ Eating the tissue cysts due to undercooked meat.

- Utilizing the food or water which is contaminated with feces of definitive host.

- Organ transplantation or blood transfusion.

- Vertical transmission to the offspring.

In humans, the cysts commonly harbor in muscles, eyes, brain and myocardium and may survive throughout the life (https://www.cdc.gov/parasites/ toxoplasmosis/ biology.html). The camel-rearing nomads in Asia and Africa usually like to eat raw camel liver (Saeed et al. 2005; Gebremedhin et al. 2014), resulting in providing the favorable conditions for transmission of $T$. gondii infection from camel to human population (Belluco et al. 2016). Additionally, the use of camel milk is becoming more popular these days, as it contains higher amount of iron and vitamin $C$ for the treatment of several diseases including tuberculosis and type-1 diabetes (Boughattas 2017). However, consumption of raw camel milk can also be a source of T. gondii infection (Medani and Mohamed 2016). Several studies have shown the presence of all the three clonal lineages (Types I, II and III) in camel milk and meat (Tavakoli et al. 2018). These three types have also been reported in infected humans (Ajzenberg et al. 2009). As the conventional labeling of $T$. gondii isolates cannot sufficiently explain the diversity of prevailing genotypes (Shwab et al. 2014), multilocus targeting PCRRFLP is necessary for understanding the transmission dynamics of Toxoplasmosis in association with their dairy products and meat. Serological tests are commonly used to study the seroprevalence of $T$. gondii infection in camels (Hamidinejat et al. 2013; Fatima et al. 2019).

\section{Trypanosomiasis}

Several Trypanosoma species, including $T$. evansi, $T$. vivax, $T$. congolense and $T$. brucei, infect camels (Roettcher et al. 1987; Dirie et al. 1989; Birhanu et al. 2015), however Trypanosoma evansi, the causative agent of "Surra", is the most prevalent parasite (Desquesnes et al. 2013). This flagellated haemoprotozoan parasite is the first pathogenic mammalian trypanosome described in the World by Griffith Evans in equids in the Dera Ismail Khan district in Pakistan (Evans 1880). Due to semi loss of T. evansi mitochondrial DNA, that occurred while its separation from T. brucei (Lai et al. 2008), this parasite can be transmitted mechanically by biting flies, hence its global distribution is beyond the limits. Trypanosoma evansi infects many domestic, as well as wild mammals in Asia, Africa and South America (Aregawi et al. 2019). The recent outbreaks of Trypanosoma infection amongst camels in France, mainland Spain and the Canary Islands depicted that this parasite can spread anywhere in the world (Gutierrez et al. 2010). In one-humped camels, this infection can cause higher morbidity and severe decline in productivity and even mortality (Sazmand et al. 2011; Sazmand et al. 2016). Camels are more prone to
Trypanosoma evansi infection as compared to other animals, including small ruminants, equids, dogs, cattle and buffaloes (Aregawi et al. 2019), but in contrary to other animal species, its economic burden in camels has not been evaluated yet (Reid 2002). Zoonotic importance of $T$. evansi infection has been reported from India, Sri Lanka, Egypt, Indonesia and Vietnam (Joshi et al. 2005; Truc et al. 2013; Van Vinh Chau et al. 2016; Sawitri et al. 2019). For many years, it was assumed that human's susceptibility to this parasite may be linked to improper amount of trypanocide apolipoprotein L1 (APOL1), which is a trypanocidal component present in human serum (Vanhollebeke et al. 2006). However, its report in a diseased person with no history of previous immunity related complication, 2 wild-type APOL1 alleles and a normal serum APOL1 concentration concluded that the parasite is zoonotic in nature (Van Vinh Chau et al. 2016).

\section{Cryptosporidiosis}

The causative agent of Cryptosporidiosis is Cryptosporidium spp. (Phylum Protozoa; Subphylum Sporozoa; Class Gregarinomorphea; Subclass Cryptogregaria; Order Cryptogregarida; Family Cryptosporidiidae), which infect almost all vertebrates including camels. Various enteric protozoan parasites, such as Eimeria spp., Cystoisospora orlovi and Sarcosystis species, develop exclusively in camels (Dubey and Schuster 2018). However, a number of scientific studies reported the camel infection via different important zoonotic parasites, including Cryptosporidium spp. (Zahedi et al. 2016). Transmission of Cryptosporidiosis is associated with ingestion of contaminated food and water, with more than 8 million people get infected with this disease annually (Ryan et al. 2018). Currently, 48 Cryptosporidium species are considered valid ( Ježková et al., 2021). In the human, more than 20 species and genotypes have been reported and its clinical manifestations are associated with gastrointestinal problems (Ježková et al. 2020). The imported subtype of Cryptosporidium parvum IlaA17G2R1, C. hominis, $C$. andersoni, Cryptosporidium rat genotype IV and Cryptosporidium camel genotype have been reported in dromedary camels through PCR and sequencing. The subtype IIaA17 $\mathrm{G}_{2} \mathrm{R} 1$ of $C$. parvum has zoonotic importance and reported from all over the World in humans and animals ( $\mathrm{Gu}$ et al. 2016; Baroudi et al. 2018; Zahedi et al. 2018; El-Alfy et al. 2019;). Although, no direct transmission of $C$. parvum and $C$. andersoni to humans has been reported in contrast to other livestock such as cattle (Lal et al. 2016), however, there is only one study from Iran which revealed the zoonotic association with camels, where $24 \%$ of the farmers were found seropositive for Cryptosporidium spp. (Sazmand et al. 2012).

\section{Echinococcosis}

Genus Echinococcus of family Taeniidae causes Echinococcosis of zoonotic importance. Echinococcus granulosus sensu lato causes Cystic Echinococcosis (CE), 
first reported from Sudan in 1908 in the annual reports of the Ministry of Animal Resources, Khartoum, Sudan (Craig et al. 1996). Echinococcus granulosus sensu lato has 10 genotypes (G1-10). Among these, most important ones are E. granulosus (sensu stricto) (genotype $\mathrm{G}_{1}-\mathrm{G}_{3}$ and their micro-variant), E. ortleppi (G5) and $E$. intermedius $\left(\mathrm{G}_{6}-\mathrm{G}_{7}\right)$ in camels (McManus and Thompson 2003; Deplazes et al. 2017; Ebrahimipour et al. 2017; Dehghani et al., 2020). Canines are the definitive hosts, while vertebrates (domestic and wild ruminants, horses, pigs, camels and member of cervid family) are the intermediate host for Echinococcosis. The disease is distributed in endemic areas of North Africa, Middle East, South and Central America, Asia and Europe. This parasite resides in the gastrointestinal tract of the final host and visceral organs of the intermediate host. The parasite forms hydatid cysts in visceral organs of the host and the disease is called as hydatidosis (Craig et al. 1996). Life cycle of this parasite starts with the release of gravid proglottids from the final host with faeces in the environment, which are ingested by intermediate hosts (ruminants, wild animals and humans). Eggs hatch in the small intestine of the intermediate host to release sixhooked oncospheres. These oncospheres penetrate the intestinal mucosa to enter the circulatory system and reach various visceral organs, especially lungs and liver. The oncospheres develop into hydatid cysts, which gradually enlarge to produce protoscolices and daughter cysts. Hydatid cysts are fluid-filled bladder like structures, which are lined by delicate parasitic membrane and often encapsulated by host fibrous tissue. There is a glycoprotein layer between organ encapsulated and the cyst to protect it from host immune response. The lining of the cyst is called as germinal epithelium, from where buds are formed which grow into brood capsule (Derbel et al. 2012). Over the time, hundreds to thousands of brood capsules are produced, which contain several inverted scolices. When a cyst ruptures, it releases protoscolices to develop into secondary cysts. Larval stage (metacestode) mostly develops in lungs and liver (Ohiolei et al. 2020). The definitive hosts ingest the infected offal of intermediate host having protoscolices which evaginate to attach with intestinal mucosa and develop into adult stage within 32-80 days (Al-Khalidi et al. 2020). At this stage, only one gravid segment is shed into the environment by each worm in a week to be the source of infection for intermediate host. Humans are the aberrant intermediate hosts and become infected by ingestion of parasite eggs (Eckert and Deplazes 2004). The most common route for transmission of Cystic Echinococcosis to human, ruminants and wild animals is by consumption of water, soil or food contaminated by stools of infected dogs (Khan et al. 2020). Other sources for infection are emigrant population, none industrial abattoirs and home slaughtering of infected animals (Seimenis 2003).

The disease has high incidence in areas where there is close association among farm animals, humans and dogs. The prevalence of hydatid cyst in lungs, liver and both organs has been reported as $77.5,3.2$ and $19.4 \%$, respectively in Pakistani camels by Anwar and Khan
(1998). Estimated prevalence of Cystic Echinococcosis in camel is $8-36 \%$ in different endemic countries (Deplazes et al. 2017; Ibrahim 2010). Variation in the prevalence of Echinococcosis has been reported such as $100 \%$ of $E$. intermedius (Cardona and Carmena 2013) and 17.0-88.4\% of E. granulosus in camels (Rostami et al., 2015). It is reported that prevalence of 11 and $88 \%$ of human Cystic Echinococcosis is due to $E$. intermedius and $E$. granulosus sensu stricto, respectively (Rojas et al., 2014). As camel is the source of milk, meat and having close contact with humans, it is the major source of disease transmission in humans. Therefore, camel strains of $E$. granulosus and E. intermedius have been detected from human infections (Sadjjadi et al., 2013). Hence, camels are the most important intermediate hosts of Echinococcosis in endemic areas of different countires, where they play an important role in the transmission of E. granulosus to humans. Yet, nature and variation of Echinococcus in the camel is not properly understood (Laurimäe et al., 2018). It is estimated that hydatid disease results in economic losses of US\$165.72 per hundred infected camels (Latif et al. 2010).

The E. granulosus (hydatidosis) is suggested to be diagnosed by identification of cyst-like mass with history of sheepdog exposure to endemic areas (Eckert and Deplazes 2004). As adults shed a gravid segment in a week, in most cases gravid segment cannot be found in the faeces of the final host. Therefore, arecoline purges are used to flush out the adult worms for diagnosis of the parasite (Varcasia et al. 2004). The anterior portion of worm is buried inside the intestinal mucosa, therefore it is very hard to observe the worm. Hence, microscopic examination of intestinal scraping is required for the detection of these worms. Several advanced imaging diagnostic techniques for Cystic Echinococcosis are CT scans, ultrasonography, and MRIs. The Cystic Echinococcosis must be differentiated from malignant and benign neoplasms, abscesses, cavitary tuberculosis, mycoses and benign cysts (Stojkovic et al. 2012). After identification of cysts by using the above technique, serological test is necessary for confirmatory diagnosis. Serologic tests, including indirect hemagglutination test and enzyme-linked immunosorbent assay (ELISA), are highly sensitive methods (Auer et al., 1988).

The treatment methods vary with cyst characteristics such as type, size, location and post-operative complications. In the past, surgery was the only option for treatment of hydrated cysts. It is more difficult to treat Alveolar Echinococcosis than Cystic Echinococcosis, the former usually requires long-term chemotherapy and radical surgery, or both at same time. Liver cysts greater than $7.5 \mathrm{~cm}$ are more likely to possess biliary communication; surgical removal may be the best option (Greco et al. 2019). Cyst puncture, chemotherapy, and PAIR (percutaneous aspiration, chemicals injection and re-aspiration) are being used instead of surgery. Few cysts do not show any symptoms (inactive) and often go away un-treated. Benzimidazoles remain effective treatment in some patients. Albendazole (10-15 $\mathrm{mg} / \mathrm{kg})$ remains effective in patients having multiple but small cysts in 
several organs. The use of mebendazole $(40-50 \mathrm{mg} / \mathrm{kg}$ ) continuously for several months has been found highly effective. Additionally, the use of both methods (chemotherapy and surgery) has been found very effective (Velasco-Tirado et al. 2018).

Control of Echinococcosis is possible by preventing transmission of the parasites (Craig et al. 1996). The measures include:

1. Prevent the dogs to feed on hydatid cyst infected carcass.

2. Control the population of stray dogs.

3. Do not consume contaminated water or food (meat, vegetables and fruit).

\section{Schistosomiasis}

Schistosomiasis is the infectious disease of human and vertebrate hosts (McManus et al. 2018). Mammalian hosts consist of humans, camels, dogs and mice (Parsani et al. 2008). The parasites reside in the vascular system (mesenteric and hepatic veins) of vertebrate hosts. Snail is the intermediate host for Schistosoma and vertebrates are the final hosts. Four Schistosoma species infect camels including $S$. bovis, $S$. mattheei, $S$. indicum and $S$. turkestanica. Except $S$. indicum, all these species have been reported in humans (Cox 2015; Sazmand et al., 2019b). The $S$. spindale has been reported in Egyptian camels (El-Khabaz et al. 2019). The disease is more common in South America, Middle East, Africa and Asia. Beside camels, more than 230 million people are affected with this disease around the globe (Colley et al. 2014). According to an estimate, more than 700 million people from more than 70 countries reside in the common disease areas. Life cycle of Schistosoma occurs in two hosts (mammals and snails). Asexual reproduction takes place in the intermediate host (snails). The development of miracidia to sporocysts occurs in the snail, the sporocysts multiply and grow into cercariae. In the final hosts (mammalian), parasites mature, mate, and reproduce to lay eggs (Viana et al. 2018). Worm eggs are released into external environment through urine or faeces of mammal hosts. These eggs transform into miracidia in freshwater, which hatch to float in water and reach the snail (Shuja et al. 2018). Daughter sporocysts either produce cercaria (cercariogenous sporocysts) or more sporocysts (Mouahid et al. 2018). Infected snails can shed hundreds of cercariae in a day (Braun et al. 2018). The parasitic larvae are released into freshwater by snails, which penetrate the skin of mammals. The disease spreads by the people having Schistosomiasis, who contaminate the freshwater with their excreta containing parasite eggs (Bekana et al., 2019). Agricultural and fishing population is more prone to Schistosomiasis.

Clinically, Schistosomiasis consists of acute and chronic forms, with incubation period of 14-84 days. Symptoms of acute disease condition include fever, headache, rashes, myalgia respiratory symptoms, chills, dermatitis and muscle aches (Sahba and Malek 1979). While, in chronic Schistosomiasis, symptoms include abdominal pain, hypertension of abdominal blood vessels, enlarged liver and spleen, blood in the urine or stool, fibrosis of urinary bladder and ureters, kidney damage and problems in passing urine (Resources for health professionals: parasites-schistosomiasis; https://www.cdc. gov/parasites /schistosomiasis/health_professionals/index.html\#tx).

Prevalence of $S$. spindale in Egyptian camel was reported as $0.8 \%$ (El-Khabaz et al. 2019). In north-west part of Thar Desert in India, prevalence was $\mathbf{1 . 4 5} \%$ (Singh et al., 2013). Similar species of Schistosoma cause disease in camels and humans. Hence, Schistosoma is zoonotically important. It is estimated that 779 million people are at the risk of infection, among them $85 \%$ are in Africa. Approximately, 207 million people from 74 countries are infected with Schistosomiasis, and 120 million of these infected people develop clinical signs of the disease (Bajiro et al. 2016).

Schistosoma eggs can be found in faecal, stool and urine which are helpful for the disease diagnosis. Several molecular techniques, such as polymerase chain reaction, nested PCR and real time PCR, are being used for molecular diagnosis of the disease. Immunological techniques, such as antibodies and/or antigens detection like immunofluorescence test (IFAT), enzyme linked immunosorbent assay (ELISA) and immunoblotting of blood or urine samples, are used for detection of the infection (Utzinger et al. 2015). The drug of choice against all species of Schistosomes is praziquantel @4omg/kg. Recovery rate of $65-90 \%$ has been found after a single dose with praziquantel. If the parasite is not killed by the drug, its efficiency of egg production is reduced by $90 \%$ (Keiser et al. 2014).

The preventive measures of Schistosomiasis are: to avoid the paddling, washing and swimming in fresh water. Preference should be given to only swim in the chlorinated or sea swimming pools. Preferably, use filtered or boiled water before drinking (Inobaya et al. 2014).

\section{Fascioliasis}

This is a food and water borne zoonotically important disease, mainly caused by Fasciola hepatica and Fasciola gigantica. The target site of Fasciola is bile duct and liver (Hanna 2015). Galba truncatula (snail) is the intermediate host of Fasciola species. F. hepatica is prevalent in almost all parts of the world, including Europe, Africa, Middle East, Asia, Oceania and parts of Latin America, while $F$. gigantica is prevalent in relatively fewer localities.

Individuals usually get infection after taking raw watercress or any water plant. After ingestion, the young worms penetrate through the intestinal mucosa, abdominal cavity, the liver and finally reach the bile duct. The parasites become adult flukes for egg production (Torgerson and Claxton 1999). Un-embryonated eggs are released into biliary ducts, and are passed in faeces/stool of humans (incidental hosts) or herbivores (definitive hosts). Embryonated eggs release miracidia in water, which swim to reach and invade the snail. In the intermediate host, the parasites undergo several developmental stages (sporocysts, rediae, and cercariae). The cercariae are released and encyst as metacercariae on 
aquatic vegetation to be exposed to the final host. Transmission occurs by ingestion of raw and freshwater vegetation containing encysted metacercariae of flukes. Usually, the invasive phase lasts for several weeks (Moazeni and Ahmadi 2016). The common symptoms are intermittent fever, abdominal pain, hepatomegaly, malaise and muscle wasting (Kaya et al. 2011). Both Fasciola species have been found in camels, with prevalence ranges from 3.3 to $15 \%$ (Banaja and Ghandour 1994; El-Khabaz et al. 2019). In Pakistan, prevalence of Fasciolosis is recorded as $30.73 \%$ in camels (Ijaz et al., 2018). Fascioliasis is considered as a highly neglected tropical disease, which infects an estimated population of 35-72 million people around the world. Microscopic examination of faeces or stool is used to observe the presence of parasites. For this purpose, more than one specimen should be examined to find the presence of parasites. The eggs are not shed immediately after infection but it needs several months. Molecular (PCR, nPCR and RT-PCR) and immunological (ELISA, IFAT, immunoblotting) methods are more sensitive for the diagnosis of Fasciola. The drug of choice for Fascioliasis is triclabendazole, which is administered orally in two doses (Gandhi et al. 2019). Most of infected people respond very well to the treatment. To date, no vaccine is available for humans and animals against Fasciola infection. Strict control measures for the sale and growth of watercress and other edible water plants are important.

\section{Blastocystis}

A complex and diverse group of heterotrophic and photosynthetic protozoa belong to phylum Stramenopila and the disease caused by its members is called intestinal Blastocystosis. This parasite usually colonizes asymptomatically in lower digestive tract of humans in different infective forms e.g., vacuolar, granular, amoeboid and cyctic (Parija and Jeremiah 2013; Besteiro 2014). It comprises 17 different sub-species (ST1-ST17) infecting 1 to 2 billion people, with the prevalence rates of 15 and $100 \%$ in developed and poorly developed countries, respectively. Blastocysts subtypes $\mathrm{ST}_{1}-\mathrm{ST} 9$ are mostly associated with human infection, however sub-types ST1, $\mathrm{ST}_{3}, \mathrm{ST}_{5}, \mathrm{ST}_{14}$ and $\mathrm{ST}_{15}$ and their mix infection are reported from camels in different parts of the World. Three sub-types i.e., ST1, $\mathrm{ST}_{3}$ and $\mathrm{ST}_{5}$ have zoonotic potential (Scanlan and Stensvold 2013; Lepczyńska et al. 2017; Mokhtar and Youssef 2018; Sazmand et al. 2019a). Transmission of this parasite occurs through oral-fecal route by ingesting contaminated food, drinking water or through direct contact. Cystic form of Blastocyst can survive up to 19 days in water under normal temperature. Infection of Blastocystis can be characterized by diarrhea, abdominal pain and irritable bowel syndrome (Garcia 2017; Toro Monjaraz et al. 2018). Blastocysts subtypes, immune level of the host and virulence of the sub-type might be the factors responsible for differences in clinical presentation (Stensvold 2013).

Diagnosis of Blastocystis is mostly made by microscopic examination of the stool, as the organism appears in stool samples in its amoebic, granular, vacuolar and cystic form. The size of $B$. hominis present in the diarrheal fluid ranges from 6 to $40 \mu \mathrm{m}$ and can be easily seen under microscope. Molecular and in-vitro culture techniques are other methods used for the identification of Blastocystis but these methods are not routinely used for diagnosis (Garcia 2006; Tan and Suresh 2006). Infection of Blastocystosis may be self-limiting and the infection should be treated symptomatically and according to the presence of other pathogens. The drug of choice for Blastocystosis is metronidazole, while trimethoprimsulfamethoxazole, nitazoxanide, and paromomycinmetronidazole may also be used in combination as an alternative, depending upon the severity of the infection (Mirza et al. 2011; Sekar and Shanthi 2013).

\section{Trichinellosis}

The disease caused by parasitic nematode Trichinella spiralis (Phylum Nematoda; Family Trichinellidae; Genus Trichinella) is called Trichinellosis which is worldwide. Trichinella spiralis life cycle's is synanthropic in pig, rat, horse, camel, dog, fox, bear, humans; muscle cysts with capsule are present (Mitreva et al. 2011). Nine species and three genotypes (T. spiralis $\left(\mathrm{T}_{1}\right), T$. native $\left(\mathrm{T}_{2}\right), T$. britovi (T3), T. pseudospiralis (T4), T. murrelli (T5), T. T6 (T6), T. nelson ( $\mathrm{T}_{7}$ ), T. T8 (T8), T. T9 (T9), T. papuae (Т10), T. zimbabwensis (T11), T. patagoniensis (T12)) of Trichinella have been documented till now; the first four $\left(\mathrm{T}_{1}-\mathrm{T}_{4}\right)$ with high pathogenicity to humans (Rostami et al. 2017), are found in the muscles of camels causing Trichinellosis in this species. Trichinellosis is cosmopolitan food borne zoonotic disease and approximately o.1 million cases are reported from all over the world annually. (https://www.cdc.gov/parasites/trichinellosis/epi.html).

The disease is more common in pork and is a food borne pathogen (Söderberg et al. 2021). Humans acquire infection by eating raw or undercooked meat of infected camels, birds, horses and pork (Bommer et al. 1980; Arbaji et al. 2005; Devleesschauwer et al. 2015). Life cycle of Trichinella spiralis consists of two phases i.e., intestinal phase and muscular phase. Infection starts by the ingestion of meat contaminated with encysted larvae. Larvae are liberated in small intestine under the influence of pepsin and gastric juices, penetrate the mucosa of small intestine and moult to adult parasites. After the period of 1-week, adult female releases larvae that migrate to striated muscles and encysted again by the host (Rostami et al. 2017; https://www.cdc.gov/parasites/ trichinellosis/biology.html). Clinical manifestation of Trichinellosis depends upon the phase of infection. In intestinal phase, the most common symptoms are diarrhea and abdominal pain, while fever, myalgia, facial oedema and myocarditis are obvious signs in muscular phase (Pozio et al. 2003; Rostami et al. 2017).

For the diagnosis of the Trichinella spp., routine examination of infected animal is mandatory. In humans, diagnosis is made by Trichinella antibody test and in some cases infected muscles biopsy is recommended. In camels, it is also diagnosed through ELISA by anti- 
Trichinella IgG. Trichinella zimbabwensis has also been identified through $5.8 \mathrm{~S}$ ribosomal RNA.

Albendazole @40o mg/kg twice up to 8 days and mebendazole $200 \mathrm{mg} / \mathrm{kg}$ thrice for three days are recommended. By adopting good hygienic conditions and avoiding improperly cooked or raw meat can also protect from the infection (Nöckler et al. 2000; https://www.cdc.gov/parasites/trichinellosis/health_prof essionals/index.html\#tx.).

\section{REFERENCES}

Al-Khalidi KAH et al., 2020. Echinococcus granulosus. In: Overview on Echinococcosis. Intech Open.

Ajzenberg D et al., 2009. Genotype of 88 Toxoplasma gondii isolates associated with toxoplasmosis in immunocompromised patients and correlation with clinical findings. Journal of Infectious Diseases 199: 1155-1167.

Anwar A and Khan M, 1998. Parasitic fauna of camel in Pakistan. In: Proceedings of the Third Annual Meeting for Animal Production under Arid Conditions, pp. 69-76.

Arbaji A et al., 2005. A 12-case outbreak of pharyngeal plague following the consumption of camel meat in north-eastern Jordan. Annals of Tropical Medicine and Parasitology 99: 789-793.

Aregawi WG et al., 2019. Systematic review and metaanalysis on the global distribution, host range, and prevalence of Trypanosoma evansi. Parasites and Vectors 12: 67.

Auer $\mathrm{H}$ et al., 1988. Combined application of enzymelinked immunosorbent assay (ELISA) and indirect haemagglutination test (IHA) as a useful tool for the diagnosis and post-operative surveillance of human alveolar and cystic echinococcosis. Zentralblatt für Bakteriologie, Mikrobiologie und Hygiene. Series A: Medical Microbiology, Infectious Diseases, Virology, Parasitology 270: 313-325.

Bahia-Oliveira L et al., 2017. Toxoplasma gondii. In: Rose JB, Jiménez-Cisneros B. Global water pathogen project. http://www.waterpathogensorg/book/toxo plasma-gondii. Accessed 27 Oct 2019.

Bajiro M et al., 2016. Prevalence of Schistosoma mansoni infection and the therapeutic efficacy of praziquantel among school children in Manna District, Jimma Zone, southwest Ethiopia. Parasites and Vectors 9: 560.

Banaja A and Ghandour A, 1994. A review of parasites of camels (Camelus dromedarius) in Saudi Arabia. JKAU Science 6: 75-86.

Baroudi D et al., 2018. Divergent Cryptosporidium parvum subtype and Enterocytozoon bieneusi genotypes in dromedary camels in Algeria. Parasitology Research 117: 905-910.

Bekana $\mathrm{T}$ et al., 2019. Transmission of Schistosoma mansoni in Yachi areas, southwestern Ethiopia: new foci. Infectious Diseases of Poverty 8: 1-8.

Belluco $\mathrm{S}$ et al., 2016. Investigating the determinants of Toxoplasma gondii prevalence in meat: a systematic review and meta-regression. PLoS One 11: eo153856.

Besteiro S, 2014. Autophagy in parasitic protists. In: Autophagy: Cancer, Other Pathologies, Inflammation, Immunity, Infection and Aging. (Elsevier), pp: 185-195.

Birhanu $\mathrm{H}$ et al., 2015. Epidemiology of Trypanosoma evansi and Trypanosoma vivax in domestic animals from selected districts of Tigray and Afar regions, Northern Ethiopia. Parasites and Vectors 8: 212.

Bommer W et al., 1980. Outbreak of Trichinelliasis in a youth centre in Neidersachsen by air-dried imported camel meat. In Proceedings of the 1st World Congress on Foodborne Infections and Intoxications, 29 June to 3 July 1980, West Berlin, Germany. (Institute of Veterinary Medicine), pp: 441-444.

Boughattas S, 2017. Toxoplasma infection and milk consumption: Meta-analysis of assumptions and evidences. Critical Reviews and Food Science Nutrition 57: 2924-2933.

Braun L et al., 2018. The effectiveness of water treatment processes against schistosome cercariae: A systematic review. PLoS Neglected Tropical Diseases 12: eooo6364.

Cardona GA and Carmena D, 2013. A review of the global prevalence, molecular epidemiology and economics of cystic echinococcosis in production animals. Veterinary Parasitology 192: 10-32.

Colley DG et al., 2014. Human Schistosomiasis. The Lancet 383: 2253-2264.

Cox FE, 2015. Taxonomy and classification of human parasitic protozoa and helminths. Manual of Clinical Microbiology 2282-2292.

Craig PS et al., 1996. Detection, screening and community epidemiology of taeniid cestode zoonoses: cystic echinococcosis, alveolar echinococcosis and neurocysticercosis. Advances in Parasitology 38: 169-250.

Deplazes P et al., 2017. Global distribution of alveolar and cystic echinococcosis. Advances in Parasitology 95: 315-493.

Derbel F et al., 2012. Hydatid cysts of the liver-diagnosis, complications and treatment. Abdominal Surgery, 5: 105-138.

Desquesnes $\mathrm{M}$ et al., 2013. Trypanosoma evansi and surra: a review and perspectives on origin, history, distribution, taxonomy, morphology, hosts, and pathogenic effects. BioMedical Research International 2013: 194176.

Devleesschauwer B et al., 2015. The low global burden of trichinellosis: evidence and implications. International Journal for Parasitology 45: 95-99.

Dirie MF et al., 1989. Camel Trypanosomiasis and its vectors in Somalia. Veterinary Parasitology 32: 285291.

Djurković-Djaković $\mathrm{O}$ et al., 2019. Toxoplasmosis: overview from a one health perspective. Food and Waterborne Parasitology 12: eooo54.

Donahoe SL et al., 2015. A review of neosporosis and pathologic findings of Neospora caninum infection in wildlife. International Journal for Parasitology: Parasites and Wildlife 4: 216-238. 
Dubey JP and Frenkel JK, 1972. Cyst-induced Toxoplasmosis in cats. Journal of Protozoology 19: 155-177.

Dubey J and Schuster R, 2018. A review of Coccidiosis in Old World camels. Veterinary Parasitology 262: 75-83.

Eckert J and Deplazes P, 2004. Biological, epidemiological, and clinical aspects of Echinococcosis, a zoonosis of increasing concern. Clinical Microbiology Reviews, 17: 107-135.

Ebrahimipour $\mathrm{M}$ et al., 2017. Molecular studies on Cystic Echinococcosis of camel (Camelus dromedarius) and report of Echinococcus ortleppi in Iran. Iranian Journal of Parasitology 12: 323.

El-Alfy ES et al., 2019. Molecular screening approach to identify protozoan and trichostrongylid parasites infecting one-humped camels (Camelus dromedarius). Acta Tropica 197: 105060.

El-Khabaz KAS et al., 2019. Protozoan and helminthes parasites endorsed by imported camels (Camel dromedarius) to Egypt. Journal of Parasitic Diseases 43: $607-615$.

Evans G, 1880. Report on 'Surra ' disease in the Dera Ismail Khan District, November 13th, 1880, Military Department, No. 493, page 4467.

Fatima $\mathrm{T}$ et al., 2019. Seroprevalence of Toxoplasma gondii in one-humped camels (Camelus dromederius) of Thal and Cholistan deserts, Punjab, Pakistan. Parasitology Research 118: 307-316.

Food Agriculture Organization of The United Nations 2019. FAOSTAT - Food and Agriculture Organization of The United Nations Statistics Division. FAO, Rome, Italy.

Gandhi P et al., 2019. Triclabendazole in the treatment of human Fascioliasis: a review. Transactions of the Royal Society of Tropical Medicine and Hygiene 113: 797-804.

Garcia LS, 2006. Diagnostic Medical Parasitology. American Society for Microbiology Press.

Garcia LS, 2017. Protozoa: Intestinal and urogenital amebae, flagellates and ciliates. In: Infectious Diseases. (Elsevier), pp. 1725-1733. e1721.

Gebremedhin EZ et al., 2014. First report of Toxoplasma gondii in camels (Camelus dromedarius) in Ethiopia: bioassay and seroepidemiological investigation. BMC Veterinary Research 10: 222.

Greco S et al., 2019. Complications of hepato echinococcosis: multimodality imaging approach. Insights Imaging 10: 113.

$\mathrm{Gu} \mathrm{Y}$ et al., 2016. Investigation on Cryptosporidium infections in wild animals in a zoo in Anhui Province. Journal of Zoo and Wildlife Medicine 47: 846-854.

Gurarie D et al., 2018. The human-snail transmission environment shapes long term Schistosomiasis control outcomes: Implications for improving the accuracy of predictive modeling. PLoS Neglected Tropical Diseases 12: eooo6514.

Gutierrez C et al., 2010. Trypanosoma evansi: Recent outbreaks in Europe. Veterinary Parasitology 174: 26-29.

Hamidinejat $\mathrm{H}$ et al., 2013. Occurrence of antiToxoplasma gondii and Neospora caninum antibodies in camels (Camelus dromedarius) in the center of Iran. Turkish Journal of Veterinary and Animal Sciences 37: 277-281.

Hanna, R, 2015. Fasciola hepatica: Histology of the reproductive organs and differential effects of triclabendazole on drug-sensitive and drug-resistant fluke isolates and on flukes from selected field cases. Pathogens 4(3): 431-456.

Ibrahim MM, 2010. Study of Cystic Echinococcosis in slaughtered animals in Al Baha region, Saudi Arabia: Interaction between some biotic and abiotic factors. Acta Tropica 113: 26-33.

Ijaz $M$ et al., 2018. Prevalence, hematology and chemotherapy of gastrointestinal helminths in camels. Pakistan Veterinary Journal, 38(1): 81-85.

Inobaya MT et al., 2014. Prevention and control of Schistosomiasis: A current perspective. Research and Reports in Tropical Medicine 2014: 65.

Ježková J et al., 2020. Cryptosporidium ratti n. sp. (Apicomplexa: Cryptosporidiidae) and genetic diversity of Cryptosporidium spp. in brown rats (Rattus norvegicus) in the Czech Republic. Parasitology 148: 84-97.

Ježková J et al., 2021. Cryptosporidium myocastoris n. sp. (Apicomplexa: Cryptosporidiidae), the species adapted to the nutria (Myocaster coypus). Microorganisms 9: 813.

Joshi PP et al., 2005. Human trypanosomiasis caused by Trypanosoma evansi in India: the first case report. American Journal of Tropical Medicine and Hygiene 73: 491-495.

Kaya $M$ et al., 2011. Clinical presentation and management of Fasciola hepatica infection: Singlecenter experience. World Journal of Gastroenterology 17: 4899 .

Keiser J et al., 2014. Praziquantel, mefloquinepraziquantel, and mefloquine-artesunatepraziquantel against Schistosoma haematobium: A randomized, exploratory, open-label trial. PLoS Neglected Tropical Diseases 8: e2975.

Khan A et al., 2020. Spread of Cystic Echinococcosis in Pakistan due to stray dogs and livestock slaughtering habits: Research priorities and public health importance. Frontiers in Public Health 7: 412.

Lal A et al., 2016. Cryptosporidiosis risk in New Zealand children under 5 years old is greatest in areas with high dairy cattle densities. EcoHealth 13: 652-66o.

Lai DH et al., 2008. Adaptations of Trypanosoma brucei to gradual loss of kinetoplast DNA: Trypanosoma equiperdum and Trypanosoma evansi are petite mutants of $T$. brucei. Proceedings of the National Academy of Sciences of the United States of America 105: 1999-2004.

Latif AA et al., 2010. Morphological and molecular characterisation of Echinococcus granulosus in livestock and humans in Punjab, Pakistan. Veterinary Parasitology 170: 44-49.

Lepczyńska $\mathrm{M}$ et al., 2017. Blastocystis: How do specific diets and human gut microbiota affect its development and pathogenicity? European Journal of 
Clinical Microbiology and Infectious Diseases 36: 15311540.

McManus D and Thompson R, 2003. Molecular epidemiology of Cystic Echinococcosis. Parasitology 127: $\mathrm{S}_{37}-\mathrm{S}_{51 .}$

McManus DP et al., 2018. Schistosomiasis. Nature Reviews Disease Primers 4: 1-19.

Medani M and Mohamed H, 2016. Camel's milk as a source of human Toxoplasmosis in Butana areaSudan. International Journal of Infectious Diseases 45: 471-472.

Mirza $\mathrm{H}$ et al., 2011. A rapid, high-throughput viability assay for Blastocystis spp. reveals metronidazole resistance and extensive subtype-dependent variations in drug susceptibilities. Antimicrobial Agents and Chemotherapy 55: 637-648.

Mitreva $\mathrm{M}$ et al., 2011. The draft genome of the parasitic nematode Trichinella spiralis. Nature Genetics 43: 228-235.

Moazeni M and Ahmadi A, 2016. Controversial aspects of the life cycle of Fasciola hepatica. Experimental Parasitology 169: 81-89.

Mokhtar A and Youssef A, 2018. Subtype analysis of Blastocystis spp. isolated from domestic mammals and poultry and its relation to transmission to their in-contact humans in Ismailia governorate, Egypt. Parasitologists United Journal 11: 90-98.

Mouahid G et al., 2018. Transplantation of schistosome sporocysts between host snails: A video guide. Wellcome Open Research 3: 3.

Nöckler K et al., 200o. Detection of Trichinella infection in food animals. Veterinary Parasitology 93: 335-350.

Ohiolei JA et al., 2020. Prevalence and distribution of Echinococcus spp. in wild and domestic animals across Africa: A systematic review and meta-analysis. Transboundary and Emerging Diseases 67: 2345-2364.

Parija SC and Jeremiah S, 2013. Blastocystis: Taxonomy, biology and virulence. Tropical Parasitology 3: 17.

Parsani et al., 2008. Common parasitic diseases of camel. Veterinary World 1: 317-318.

Pozio E et al., 2003. Clinical aspects, diagnosis and treatment of Trichinellosis. Expert Review of Antiinfective Therapy 1: 471-482.

Reid SA, 2002. Trypanosoma evansi control and containment in Australasia. Trends in Parasitology 18: 219-224.

Roettcher D et al., 1987. Trypanosomiasis in the camel (Camelus dromedarius). Revue Scientifique et Technique 6: 463-470.

Rojas CAA et al., 2014. Echinococcus granulosus sensu lato genotypes infecting humans-review of current knowledge. International Journal of Parasitology 44: $9^{-18 .}$

Rostami S et al., 2015. Genetic characterization of Echinococcus granulosus from a large number of formalin-fixed, paraffin-embedded tissue samples of human isolates in Iran. American Journal of Tropical Medicine and Hygiene 92(3): 588-594.

Rostami A et al., 2017. Meat sources of infection for outbreaks of human Trichinellosis. Food
Microbiology 64: 65-71.

Ryan U et al., 2018. Foodborne Cryptosporidiosis. International Journal for Parasitology 48: 1-12.

Sadjjadi et al., 2013. Evidence that the Echinococcus granulosus G6 genotype has an affinity for the brain in humans. International Journal of Parasitology 43(11): 875-877.

Saeed $A A B$ et al., 2005. Plague from eating raw camel liver. Emerging Infectious Diseases 11: 1456-1457.

Sahba GH and Malek EA, 1979. Dermatitis caused by cercariae of Orientobilharzia turkestanicum in the Caspian Sea area of Iran. The American Journal of Tropical Medicine and Hygiene 28: 912-913.

Sawitri DW et al., 2019. Detection of Surra (Trypanosomiasis) positivity in humans in an outbreak area of Indonesia. Medical Journal of Indonesia 28: 196-202.

Sazmand A and Joachim A, 2017. Parasitic diseases of camels in Iran (1931-2017)- a literature review. Parasite 24: 21.

Sazmand A et al., 2011. Serobiochemical alternations in subclinically affected dromedary camels with Trypanosoma evansi in Iran. Pakistan Veterinary Journal 31: 223-226.

Sazmand A et al., 2012. Prevalence of Cryptosporidium spp. in camels and involved people in Yazd Province, Iran. Iranian Journal of Parasitology 7: 8o-84.

Sazmand A et al., 2016. Molecular identification of hemoprotozoan parasites in camels (Camelus dromedarius) of Iran. Iranian Journal of Parasitology 11: 568-573.

Sazmand A et al., 2019a. Vector-borne bacteria in blood of camels in Iran: New data and literature review. Comparative Immunology, Microbiology and Infectious Diseases 65: 48-53.

Sazmand A et al., 2019b. Zoonotic parasites of dromedary camels: So important, so ignored. Parasites and Vectors 12: 610.

Scallan E et al., 2015. An assessment of the human health impact of seven leading foodborne pathogens in the United States using disability adjusted life years. Epidemiology and Infection 143: 2795-2804.

Scanlan PD and Stensvold CR. 2013. Blastocystis: getting to grips with our guileful guest. Trends in Parasitology 29: 523-529.

Schuster RK, 2018. Parasites of dromedaries and bactrian camels-a review part 1: Stenoxenous parasites. Journal of Camel Practice and Research 25: 1-8.

Seimenis A, 2003. Overview of the epidemiological situation on Echinococcosis in the Mediterranean region. Acta Tropica 85(2): 191-195.

Sekar U and Shanthi M, 2013. Blastocystis: Consensus of treatment and controversies. Tropical Parasitology 3: 35 .

Shuja A et al., 2018. Intestinal Schistosomiasis: A rare cause of abdominal pain and weight loss. 10(1): e2o86.

Shwab EK et al., 2014. Geographical patterns of Toxoplasma gondii genetic diversity revealed by multilocus PCR-RFLP genotyping. Parasitology 141: 453-461. 
Singh $\mathrm{T}$ et al., 2013. Hepatic Schistosomiasis in camel (Camelus dromedarius). Comparative Clinical Pathology 22: 989-991.

Söderberg R et al., 2021. Low prevalence of Cysticercosis and Trichinella infection in pigs in rural Cambodia. Tropical Medicine and Infectious Disease 6(2): 100.

Stensvold CR, 2013. Blastocystis: genetic diversity and molecular methods for diagnosis and epidemiology. Tropical Parasitology 3: 26.

Stojkovic M et al., 2012. Diagnosing and staging of Cystic Echinococcosis: How do CT and MRI perform in comparison to ultrasound?. PLoS Neglected Tropical Diseases 6: e1880.

Tan T and Suresh K, 20o6. Predominance of amoeboid forms of Blastocystis hominis in isolates from symptomatic patients. Parasitology Research 98: 189193.

Tavakoli A et al., 2018. The first survey of isolation and molecular typing of Toxoplasma gondii by bioassay and PCR method in BALB/c mice in camels from eastern Iran. Iranian Journal of Parasitology 13: 382391.

Torgerson P and Claxton J, 1999. Epidemiology and control. In: Dalton EJP (ed) Fasciolosis. CABI Publishing, Wallingford.

Toro Monjaraz EM et al., 2018. Blastocystis hominis and chronic abdominal pain in children: Is there an association between them? Journal of Tropical Pediatrics 64: 279-283.

Truc $\mathrm{P}$ et al., 2013. Atypical human infections by animal trypanosomes. PLoS Neglected Tropical Diseases 7: e2256.
Utzinger $\mathrm{J}$ et al., 2015. New diagnostic tools in Schistosomiasis. Clinical Microbiology and Infection 21: 529-542.

Chau NVV et al., 2016. A clinical and epidemiological investigation of the first reported human infection with the zoonotic parasite Trypanosoma evansi in Southeast Asia. Clinical Infectious Diseases 62: 10021008.

Vanhollebeke B et al., 2006. Human Trypanosoma evansi infection linked to a lack of Apolipoprotein L-I. New England Journal of Medicine 355: 2752-2756.

Varcasia A et al., 2004. The diagnosis of Echinococcus granulosus in dogs. Parassitologia 46: 409-412.

Velasco-Tirado V et al., 2018. Medical treatment of Cystic Echinococcosis: Systematic review and meta-analysis. BMC Infectious Diseases, 18: 1-19.

Viana $M$ et al., 2018. The effects of subcurative praziquantel treatment on life-history traits and trade-offs in drug-resistant Schistosoma mansoni. Evolutionary Applications 11: 488-500.

Wernery U et al., 2014. Camelid Infectious Disorders. OIE (World Organisation for Animal Health), Paris, France, p 500.

Zahedi A et al., 2016. Public health significance of zoonotic Cryptosporidium species in wildlife: Critical insights into better drinking water management. International Journal for Parasitology: Parasites and Wildlife 5: 88-109.

Zahedi A et al., 2018. First report of Cryptosporidium parvum in a dromedary camel calf from Western Australia. Acta Parasitologica 63: 422-427.

Zhu S et al., 2019. A review of zoonotic pathogens of dromedary camels. EcoHealth 16: 356-377. 